\title{
NOVEL MULTIPLIERLESS WIDEBAND COMB COMPENSATOR WITH HIGH COMPENSATION CAPABILITY
}

\author{
Gordana Jovanovic Dolecek ${ }^{1}$, Ricardo Garcia Baez ${ }^{1}$, Gerardo Molina Salgado ${ }^{1}$, and Jose \\ de la Rosa ${ }^{2}$ \\ ${ }^{1}$ Department of Electronics, Institute INAOE Puebla, Mexico \\ ${ }^{2}$ Institute IMSE-CNM (CSIC), Sevilla, Spain \\ Emails: gordana@ieee.org; jrgarciabaez@gmail.com, \\ gerardo.molina.salgado@gmail.com; jrosa@imse-cnm.csic.es
}

\begin{abstract}
This paper proposes a novel multiplierless comb compensation filter, which has the absolute passband deviation less than $0.1 \mathrm{~dB}$ in the wide passband. The compensator consists of a cascade of two simple filter sections, both operating at a low rate. The magnitude characteristics of the two component filters are synthesized as sinewave functions, in which the main design parameters correspond to the amplitudes of sinewave functions. A systematic procedure is followed to select synthesis parameters, which depend only on the number of cascaded comb filters. In particular, they are independent of the decimation factor. Comparisons with comb compensators from literature illustrate the benefits of the proposed design.
\end{abstract}

Key words: Decimation; Aliasing; Comb filter; Passband droop; Compensator; Sinewave functions.

\section{INTRODUCTION}

Decimation is the process of decreasing sampling rate in a digital form which has applications in communications, audio signal processing, Sigma Delta Analog to Digital converters, among others [1]. However, this process introduces aliasing which must be eliminated by a decimation filter. The most simple decimation filter is the comb filter, 
usually used at the first decimation stage. The system function of the comb is given as [2]:

$$
H(z)=\left[\frac{1}{M} \frac{1-z^{M}}{1-z^{-1}}\right]^{K},
$$

where $M$ is the decimation factor and $K$ is the number of comb sections connected in series.

The wide passband of a comb filter is defined by the edge frequency, given by:

$$
\omega_{p}=\frac{\pi}{2 M}
$$

In order to achieve correct performance, the comb decimation filter should have a flat magnitude response in the signal bandwidth of interest. However, it is well known that the magnitude characteristic of comb filter,

$$
\left|H\left(e^{j \omega}\right)\right|=\left|\frac{1}{M} \frac{\sin (\omega M / 2)}{\sin (\omega / 2)}\right|^{K},
$$

exhibits a high passband droop, which increases with the number of the cascaded comb filters $[1,2]$.

In order to address this limitation, different compensators have been proposed to reduce the passband droop of the wide passband of comb-based decimation structures [3-12]. In the majority of the cases, proposed compensators require multipliers for their implementation [6], [7]. However, as comb structures can be synthesized as multiplierless filters, their associated compensators should be also implemented without multipliers. In addition, like CIC (Cascaded-Integrators-Combs) structure, the design of the 
compensator should not be redesigned every time the values of design parameters $M$ and $K$ are modified.

This paper addresses the above limitations and proposes a novel structure for comb compensator with the following main features:

- The passband edge frequency is defined as $\pi /(2 M)$.

- The compensator design is valid for a decimation factor $M>10$.

- Multiplierless filter structure

- Filter design is defined only by the comb design parameter, $K$.

- Filter that has the passband absolute deviation less than $0.1 \mathrm{~dB}$.

- Design approach has less complexity than previous approaches with the similar absolute passband deviation.

The paper is organized as follows. Next Section describes the proposed compensator structure. Sections 3 and 4 present the methodology followed to properly select the main design parameters of the proposed compensator structure. Section 5 provides comparisons of the presented filter against previous approaches.

\section{DESCRIPTION OF PROPOSED FILTER}

\subsection{Sinewave-based magnitude responses}

The magnitude response of the compensator under study has to be approximately equal to the inverse of the magnitude response of a comb-based filter. The latter can be implemented as a squared sinewave-based magnitude response, given by [3]: 


$$
\left|G_{2}\left(e^{j \omega M}\right)\right|=\left|1+B_{2} \sin ^{2}(\omega M / 2)\right|=1+B_{2} \sin ^{2}(\omega M / 2),
$$

where $B_{2}$ is the sinewave amplitude and $M$ is the decimation factor.

It can be shown from (4) that the absolute passband deviation depends on the design parameter, $B_{2}$, which in turns depends on the comb-filter parameter, $K$. Indeed, as demonstrated in [3], the maximum absolute deviation, $\delta$, lower than $0.4 \mathrm{~dB}$ can be obtained for $K=1,2, \ldots, 6$.

In order to achieve better approximation of the inverse of the comb magnitude response, a filter structure with the transfer function given in (4) can be connected in cascade with a stage having a fourth-order sine-based transfer function given by:

$$
\left|G_{1}\left(e^{j \omega M}\right)\right|=\left|1+B_{1} \sin ^{4}(\omega M / 2)\right|=1+B_{1} \sin ^{4}(\omega M / 2),
$$

where $B_{1}$ is amplitude of the sine-based responses.

As a result, the proposed compensator structure has the following magnitude response:

$$
\left|G_{c}\left(e^{j \omega M}\right)\right|=\left[1+B_{1} \sin ^{4}(\omega M / 2)\right]\left[1+B_{2} \sin ^{2}(\omega M / 2)\right] .
$$

An example is provided in Fig.1(a), where the gain response of the inverse comb filter along with the gain responses from (4) and (5) are shown, with values $B_{1}=1$, and $B_{2}=0.75$. It can be observed that both the expressions in (4) and (5) approximate the inverse of the comb gain response reasonably well, with the expression in (5) offering a better approximation.

Fig.2(b) shows the gain responses of the inverse comb filter and filter (6), using the same amplitudes $B_{1}=1$, and $B_{2}=0.75$ as in Fig.1(a). It can be noticed that the proposed compensator (6) matches well with the inverse of the comb magnitude characteristic. 
Thus, by combining sine-based magnitude responses (4) and (5), a better inverse comb magnitude response approximation can be achieved than using each one separately. Based on this observation, a proper selection of $B_{1}$ and $B_{2}$ could offer the best approximation for given values of $K$ and $M$, i.e., the main design parameters of a combbased structure.

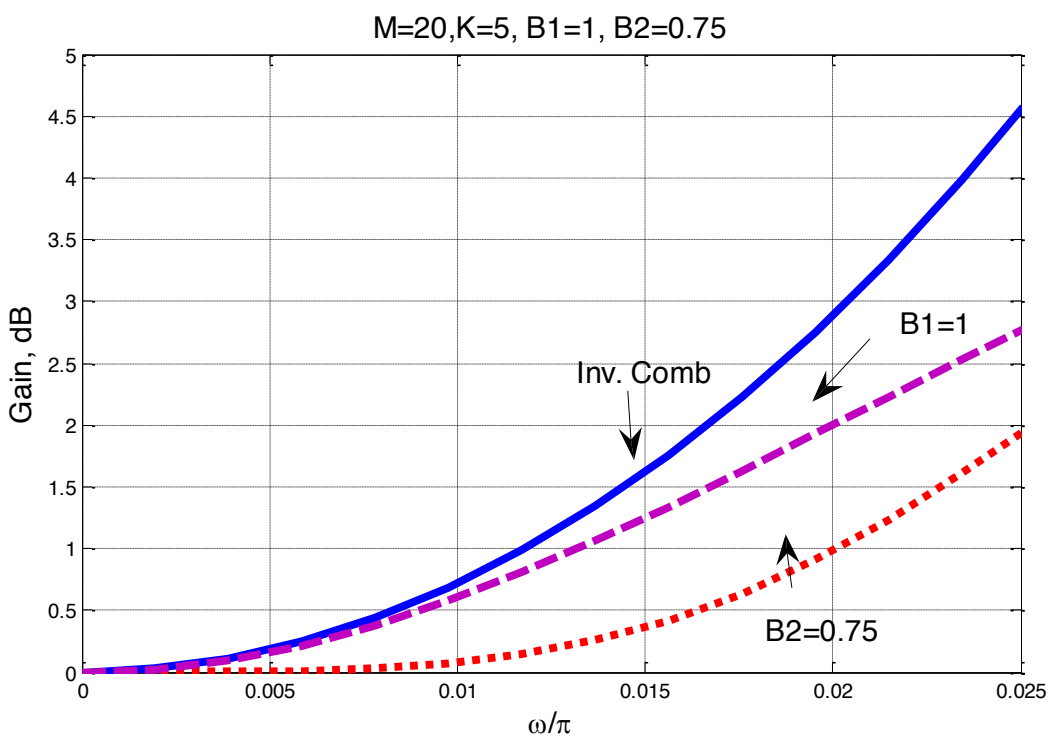

(a) Inverse comb magnitude response, $G_{1}\left(e^{j w M}\right)$ with $B_{1}=1, G_{2}\left(e^{j w M}\right)$ with $B_{2}=0.75$.

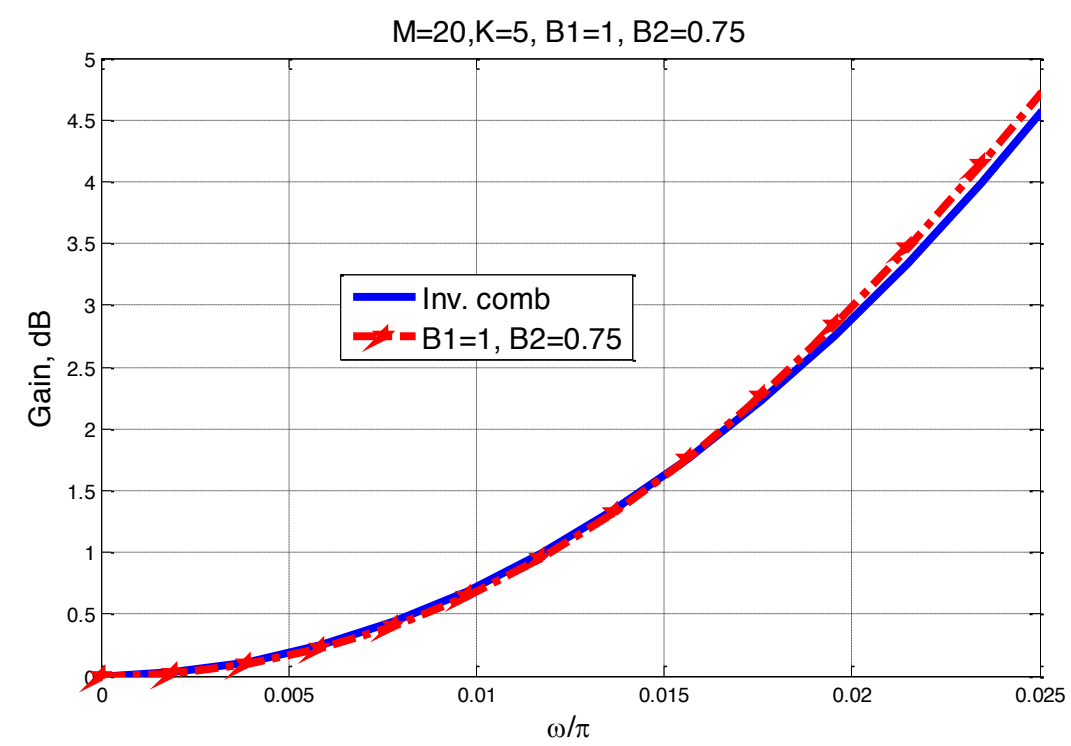

(b)Inverse comb magnitude response and $G_{\mathrm{c}}\left(e^{j w M}\right)$ with $B_{1}=1$, and $B_{2}=0.75$.

Fig.1. Approximation of inverse comb magnitude characteristic with sine-based responses. 


\subsection{Proposed structures}

The transfer function of (4) can be expressed in the $z$-domain as [3]:

$$
G_{2}\left(z^{M}\right)=2^{-2}\left[-B_{2}+\left(2^{2}+2 B_{2}\right) z^{-M}-B_{2} z^{-2 M}\right] .
$$

This function can be decimated by a factor of $M$ to lower rate and written as [3]:

$$
G_{2}(z)=2^{-2}\left[\left(-1+2 z^{-1}-z^{-2}\right) B_{2}+2^{2} z^{-1}\right] .
$$

The transfer function given in (8) can be implemented by using the structure depicted in Fig. 2(a), which requires three adders and one multiplier, (coefficient $B_{2}$ ).

Let us now turn our attention to the transfer function of the filter given in (5). By using the following well-known trigonometric relation:

$$
\sin ^{4}(\alpha)=[\cos (4 \alpha)-4 \cos (2 \alpha)+3] / 8,
$$

it is straightforward to rewrite the magnitude response of (5) as:

$$
\begin{aligned}
& \left|G_{1}(\omega)\right|=1+B_{1} \sin ^{4}(\omega M / 2)= \\
& 1+2^{-3} B_{1}[\cos (4 \omega M / 2)-4 \cos (2 \omega M / 2)+3]= \\
& 1+2^{-3} B_{1}[\cos (2 \omega M)-4 \cos (\omega M)+3]
\end{aligned} .
$$

By using the Euler's formula, the expression in (10) can be rewritten as:

$$
G_{1}\left(e^{j \omega M}\right)=2^{-4} B_{1}\left[1+e^{-j 4 \omega M}-4\left(e^{-j \omega M}+e^{-j 3 \omega M}\right)\right]+\left[2^{-3} B_{1} 3+1\right] e^{-j 2 \omega M} .
$$

Substituting $z=e^{j \omega}$ in (11), yields:

$$
G_{1}\left(z^{M}\right)=2^{-4} B_{1}\left[1+z^{-4 M}-4\left(z^{-M}+z^{-3 M}\right)\right]+\left[2^{-3} 3 B_{1}+1\right] z^{-2 M} .
$$

At a low rate, after applying a decimation of $M$, the expression in (12) is given by:

$$
\begin{aligned}
& G_{1}(z)= \\
& 2^{-4} B_{1}\left[1+z^{-4}-4\left(z^{-1}+z^{-3}\right)\right]+\left[2^{-3} B_{1} 3+1\right] z^{-2}= \\
& 2^{-4} B_{1}\left[1+z^{-4}-4\left(z^{-1}+z^{-3}\right)+\left(2^{2}+2\right) z^{-2}\right]+z^{-2}
\end{aligned}
$$

The transfer function given in (13) can be implemented using the structure shown in 
Fig. 2(b), which includes six adders and one multiplier, $B_{1}$.

The block diagram of the proposed compensated comb structure is shown in Fig. 2(c). Note that the proposed compensator - which consists of the cascade connection of the structures shown in Fig. 2(a) and Fig. 2(b) - is placed after the decimator, so that it operates at the lowest rate.

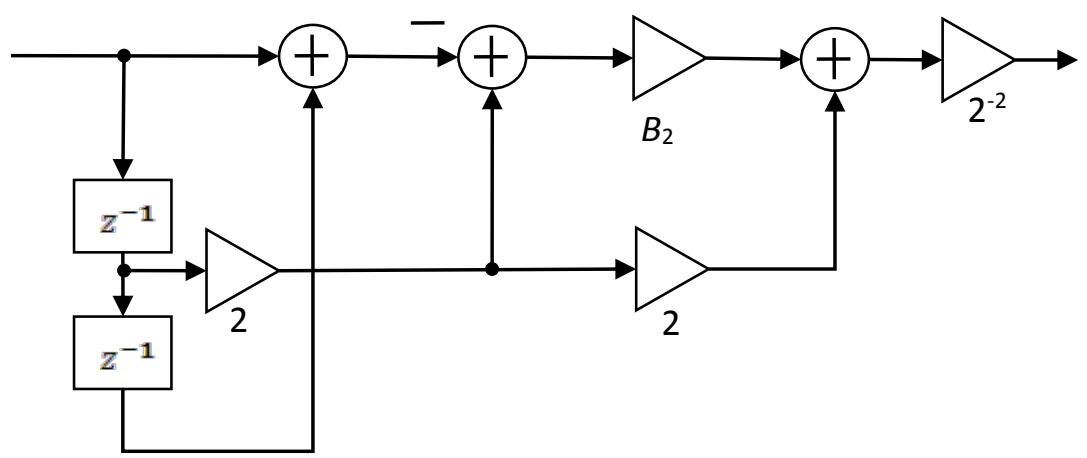

(a) Structure for the filter $G_{2}(z)$.

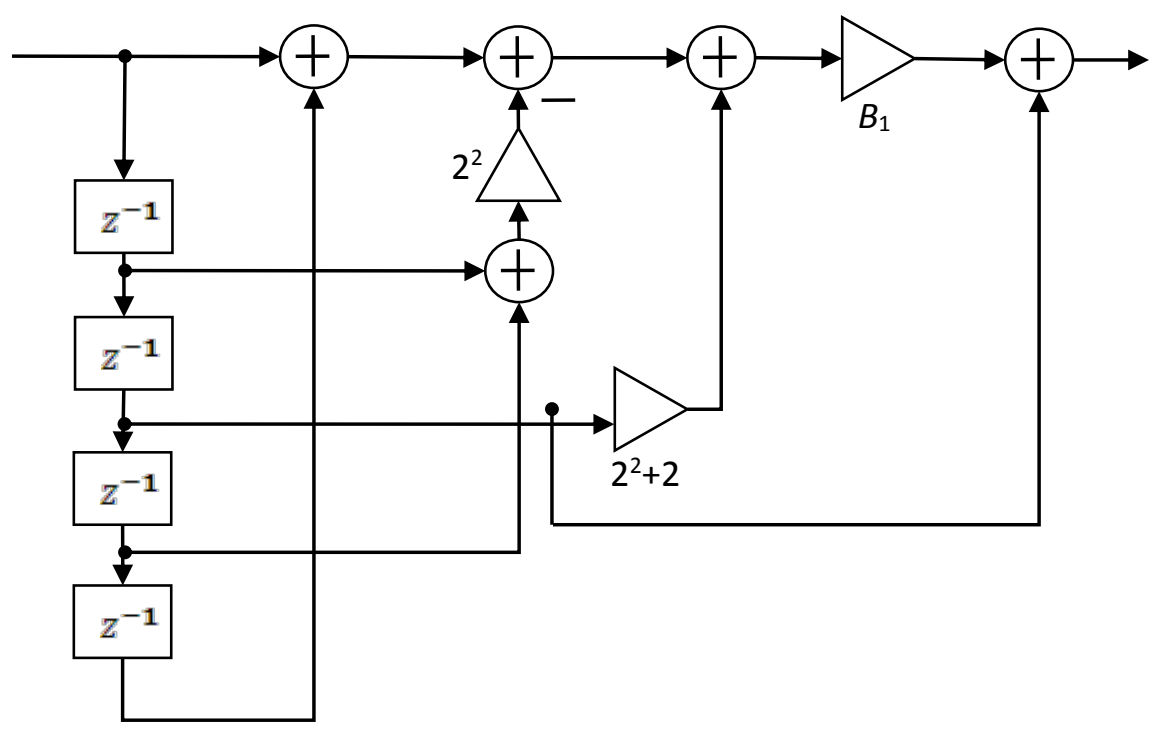

(b) Structure of the filter $G_{1}(z)$. 
(c) Comb and proposed decimator.

Fig.2. Proposed structures.

\section{PRELIMINARY DETERMINATION OF PARAMETERS}

In this section the parameters $B_{1}$ and $B_{2}$ are determined taking two conventionally chosen frequencies in the passband, $\omega_{1}$ and $\omega_{2}$.

The compensated comb magnitude response can be derived from (3) and (6) as:

$$
\left|H_{c}\left(e^{j \omega}\right)\right|=\left|H\left(e^{j \omega}\right) G_{1}\left(e^{j M \omega}\right) G_{2}\left(e^{j M \omega}\right)\right|,
$$

where $G_{1}\left(e^{j \omega M}\right)$ and $G_{2}\left(e^{j \omega M}\right)$ are given by (4) and (5), respectively.

The magnitude response of the compensated comb structure must be approximately flat in the passband, which can be formulated as:

$$
\left|H_{c}\left(e^{j \omega}\right)\right| \approx 1 ; \quad 0 \leq \omega \leq \omega_{p} .
$$

In order to find the values of $B_{1}$ and $B_{2}$ that achieve the maximum compensation, the expression in (15) is evaluated at two frequencies $\omega_{1}$ and $\omega_{2}$, which are within the passband, so that $\left|H_{C}\left(e^{j \omega 1}\right)\right| \approx 1$ and $\left|H_{C}\left(e^{j \omega 2}\right)\right| \approx 1$. We denote the values $B_{1}$ and $B_{2}$ as $B_{1 \mathrm{~s}}$ and $B_{2 \mathrm{~s}}$ because their choice is related with the specified frequencies in the passband, $\omega_{1}$ and $\omega_{2}$

\subsection{Determination of $\boldsymbol{B}_{2 s}$}

Without loss of generality, the frequency $\omega_{1}$ is chosen for convenience in such a way that the fourth power of the sine function in (5) is approximately zero, thus resulting in:

$$
\left|G_{1}\left(e^{j \omega_{1} M}\right)\right| \approx 1 .
$$


This way, evaluating (15) at $\omega_{1}$ and combing with (16), we have:

$$
\left|H\left(e^{j \omega_{1}}\right) G_{2}\left(e^{j M \omega_{1}}\right)\right| \approx 1 .
$$

Therefore,

$$
\left|G_{2}\left(e^{j M \omega_{1}}\right)\right| \approx \frac{1}{\left|H\left(e^{j \omega_{1}}\right)\right|}
$$

Using (4) and (1), and using the fact that the magnitude response of a comb filter is always positive in the passband, $0 \leq \omega \leq \omega_{P}$, the approximation in (18) can be expressed as:

$$
\left[1+B_{2 s} \sin ^{2}\left(\omega_{1} M / 2\right)\right] \approx\left[\frac{M \sin \left(\omega_{1} / 2\right)}{\sin \left(\omega_{1} M / 2\right)}\right]^{K}
$$

Thus, $B_{2 \mathrm{~s}}$ can be derived from (19):

$$
B_{2 s} \approx \frac{-1+\left[\frac{M \sin \left(\omega_{1} / 2\right)}{\sin \left(\omega_{1} M / 2\right)}\right]}{\sin ^{2}\left(\omega_{1} M / 2\right)} .
$$

As an example, taking $\omega_{1}=\omega_{P} / 4$, which satisfy (16), we have:

$$
B_{2 s} \approx \frac{-1+\left[\frac{M \sin (\pi /(16 M))}{\sin (\pi / 16)}\right]}{\sin ^{2}(\pi / 16)} .
$$

Note that the choice of $B_{2 s}$ depends on $M$. Assuming a sufficiently large value of $M$, for instance $M>10$, the following approximation is valid:

$$
\sin (\pi /(16 M)) \approx \pi /(16 M),
$$

and hence, $B_{2 \mathrm{~s}}$ simplifies to:

$$
B_{2 s} \approx \frac{-1+\left[\frac{\pi / 16}{\sin (\pi / 16)}\right]^{K}}{\sin ^{2}(\pi / 16)},
$$


which depends only on the value of $K$.

\subsection{Determination of $B_{1}$}

Proceeding in a similar way, and evaluating (15) at frequency $\omega_{2}$ it can be shown that:

$$
\left[1+B_{1 s} \sin ^{4}\left(\omega_{2} M / 2\right)\right]\left[1+B_{2 s} \sin ^{2}\left(\omega_{2} M / 2\right] \approx\left[\frac{\omega_{2} M / 2}{\sin \left(\omega_{2} M / 2\right)}\right]^{K} .\right.
$$

The approximate expression for $B_{1 \mathrm{~s}}$ can be derived from (24), giving:

$$
B_{1 s} \approx \frac{-1+\frac{\left[\frac{M \omega_{2} / 2}{\sin \left(M \omega_{2} / 2\right)}\right]^{K}}{1+B_{2 s} \sin ^{2}\left(M \omega_{2} / 2\right)}}{\sin ^{4}\left(M \omega_{2} / 2\right)} .
$$

Assuming that $M>10$ and $\omega_{2}=\omega_{P}$ the expression in (25) can be simplified as:

$$
B_{1 s} \approx \frac{-1+\frac{\left[\frac{\pi / 4}{\sin (\pi / 4)}\right]^{K}}{1+B_{2 s} \sin ^{2}(\pi / 4)}}{\sin ^{4}(\pi / 4)} .
$$

Note that the parameters $B_{1 \mathrm{~s}}$ and $B_{2 \mathrm{~s}}$ do not depend on $M$, provided that $M \geq 10$, and depend only on the comb parameter $K$. As an illustration, Table 1 shows the values of $B_{1 \mathrm{~s}}$ and $B_{2 \mathrm{~s}}$ for different values of $K$, considering $\omega_{1}=\omega_{P} / 4$ and $\omega_{2}=\omega_{P}$.

Table 1: The values of $B_{1 \mathrm{~s}}$ and $B_{2 \mathrm{~s}}$ for $\omega_{1}=\omega_{P} / 4$ and $\omega_{2}=\omega_{P}$

\begin{tabular}{|l|l|l|}
\hline$K$ & $B_{1 \mathrm{~s}}$ & $B_{2 \mathrm{~s}}$ \\
\hline 6 & 0.9510 & 1.0341 \\
\hline 5 & 0.7305 & 0.8590 \\
\hline 4 & 0.5350 & 0.6849 \\
\hline 3 & 0.3639 & 0.5121 \\
\hline 2 & 0.2173 & 0.3403 \\
\hline 1 & 0.0956 & 0.1696 \\
\hline
\end{tabular}


In following example we illustrate that the same compensator parameters $B_{1 \mathrm{~s}}$ and $B_{2 \mathrm{~s}}$ can be used to compensate combs with equal $K$, and different values of $M$.

\section{Example 1:}

Let us consider two comb filters with $K=4$ and two values of $M, 24$ and 18, respectively. From Table 1 the compensator parameters for both combs are: $B_{1 \mathrm{~s}}=0.535$, $B_{2 \mathrm{~s}}=0.6849$. The gain responses along with the passband and first folding band zooms are shown in Fig.3, considering $M=24$ and $M=18$. Note that the proposed filter structure provides good compensation in both cases and that the alias rejection of the comb filter is not significantly degraded due to the compensator.

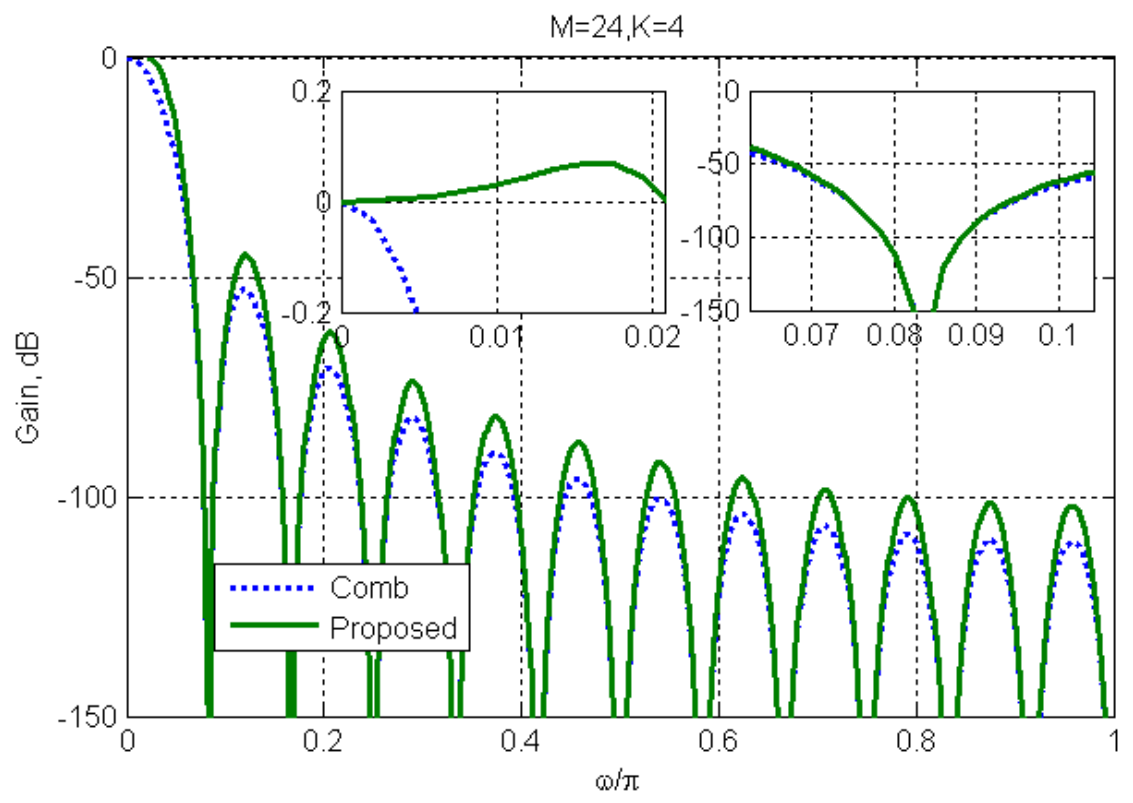

(a) $M=24, K=4$. 


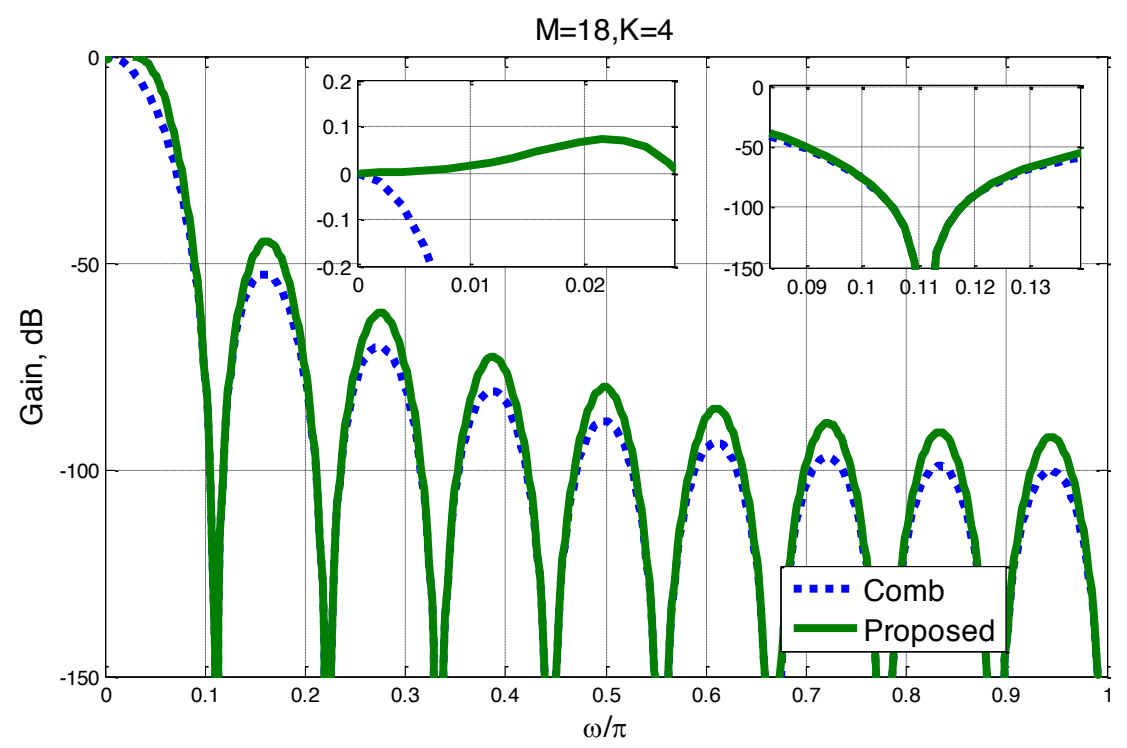

(b) $M=18, K=4$.

Fig. 3. Illustrating the compensation of comb structures with different values of $M$ and an equal value of $K$, using compensator with two multipliers.

However, in spite of the mentioned benefits, the proposed methodology presents the following limitations:

- The choice of frequencies $\omega_{1}$ and $\omega_{2}$ is arbitrary and hence, each new choice would result in different values of $B_{1 \mathrm{~s}}$ and $B_{2 \mathrm{~s}}$.

- The maximum absolute passband deviation of the compensated comb filter is not defined.

- The compensator structure requires two multipliers.

\section{DETERMINATION OF PARAMETERS $B_{1}$ AND $B_{2}$ FOR MULTIPLIERLESS COMPENSATOR}

In order to address the aforementioned limitations, our goal here is to obtain the multiplierless compensator which depends only on the comb parameter $K$, i.e. that the parameters $B_{1}$ and $B_{2}$ depend only on $K$. To this end, the proposed compensator structure is transformed into a multiplierless topology, by modifying $B_{1 \mathrm{~s}}$ and $B_{2 \mathrm{~s}}$, so that these 
coefficients can be realized by only using adders.

The values $B_{1}$ are chosen first, in the most simple form, because the subfilter $G_{1}(z)$ is more complex than the subfilter $G_{2}(z)$. Next, the values of $B_{2}$ are obtained in such way that the resulting absolute value of the passband deviation is less than the specified value $\left|\delta_{d}\right|$

With this in order, let us first consider the estimated values of $B_{1 \mathrm{~s}}$, presented in Table 1 . These values are taken as a starting point to represent the filter parameters in the form of a power of two. This can be done by rounding the values of $B_{1 \mathrm{~s}}$, resulting in the values shown in Table 2 and denoted as $B_{1}$. Note that coefficient $B_{1}$ does not introduce any adders in the proposed compensator structures.

The modified values of $B_{2 \mathrm{~s}}$, denoted as $B^{\prime}{ }_{2}$, are obtained using a similar procedure as the one proposed in [3]. Let us define the absolute value of maximum passband deviation as $\left|\delta_{d}\right|$, so that the following condition is imposed

$$
20 \log _{10}\left|H_{c}\left(e^{j \omega_{k}}\right)\right|<\left|\delta_{d}\right| / 2[\mathrm{~dB}]
$$

where $\omega_{k}$ are arbitrary frequencies in the passband, given by

$$
\omega_{k}=\frac{\pi k}{2 M N} ; k=1, \ldots N
$$

Note, that, since the rounding of coefficients introduces magnitude response deviation, a stronger condition in (28) has been used, taking $\mid \delta_{d} / / 2$. This way, substituting (28) into (27), $N$ different values for $B_{2 \mathrm{~s}}$ can be determined, denoted as $B_{2 s k}$, and given by:

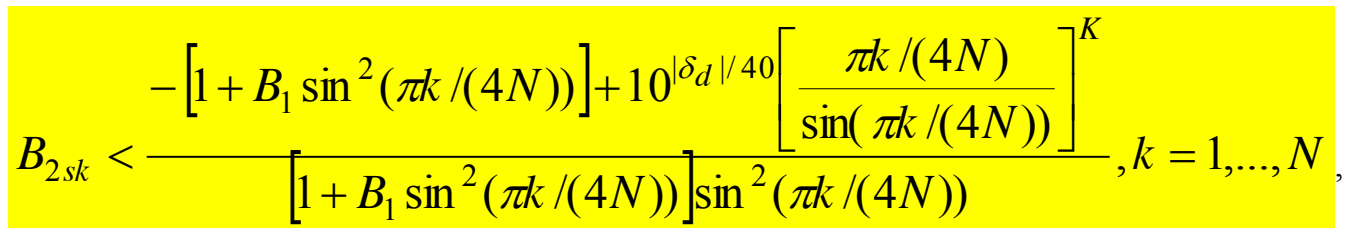


where the values $B_{1}$ are given in Table 2 .

The derivation of (29) is provided in Appendix in more detail.

Thus, for a given value of $K$, and the $N$ values of $B_{2 s k}$ obtained from (29), the values of $B_{2}{ }_{2}$, are computed as:

$$
B_{2}^{\prime}=\min _{k}\left\{B_{2 s k}\right\}, k=1, \ldots, N
$$

which correspond to the minimum of all possible values of $B_{2 \text { sk }}$ according to (30).

The expression in (30) can be easily implemented in Matlab scripts for given values of $K$, and $B_{1}$, and considering $\left|\delta_{d}\right|=0.1$. This value of $\left|\delta_{d}\right|=0.1$ is evaluated by Matlab simulations and is chosen as a reasonable value, (by considering that the magnitude response of the proposed compensator structure can only approximate that of the corresponding inverse comb) to ensure that the maximum passband deviation is always less than $0.1 \mathrm{~dB}$ for $M>10$ and $K=1, \ldots, 6$.

Based on these considerations, this maximum passband deviation of $0.1 \mathrm{~dB}$ has been chosen to obtain the $B_{2}^{\prime}$ values shown in the third column of Table 2.

Table 2: Values of $B_{1}$ and $B{ }^{\prime}{ }_{2}$.

\begin{tabular}{|l|l|l|}
\hline$K$ & $B_{1}$ & ${ }^{\prime}{ }_{2}$ \\
\hline 6 & 1 & 0.9858 \\
\hline 5 & 1 & 0.7188 \\
\hline 4 & $1 / 2$ & 0.6905 \\
\hline 3 & $1 / 2$ & 0.45 \\
\hline 2 & $1 / 4$ & 0.3281 \\
\hline 1 & 0 & 0.2129 \\
\hline
\end{tabular}

Finally, the values of $B^{\prime}{ }_{2}$, obtained from (30) are rounded, resulting in the coefficients denoted as $B_{2}$, which can be expressed in the form of sums of power of two as shown in 
Table 3. This table shows also the values of $B_{1}$ and the number of adders required to implement each coefficient in the proposed compensator.

Table 3: The values $B_{1 r}, B_{2 r}$, and the number of adders.for $\left|\delta_{d}\right|=0.1 \mathrm{~dB}$

\begin{tabular}{|l|l|l|l|}
\hline$K$ & $B_{1}$ & $B_{2}$ & $\begin{array}{l}\text { Number of } \\
\text { adders }\end{array}$ \\
\hline 6 & 1 & $2^{0}-2^{-6}$ & 10 \\
\hline 5 & 1 & $2^{0}-2^{-2}-2^{-5}$ & 11 \\
\hline 4 & $2^{-1}$ & $2^{-1}+2^{-3}+2^{-4}$ & 11 \\
\hline 3 & $2^{-1}$ & $2^{-1}-2^{-4}$ & 10 \\
\hline 2 & $2^{-2}$ & $2^{-2}+2^{-4}$ & 10 \\
\hline 1 & 0 & $2^{-2}-2^{-5}$ & 4 \\
\hline
\end{tabular}

In general, the proposed compensator requires no more than 11 adders, providing the maximum absolute passband deviation lower than $0.1 \mathrm{~dB}$.

\section{Example 2:}

Analogously, for the multiplier-based compensator, the proposed multiplierless compensator can also be used for different values of $M$, for the same value of parameter $K$. As an example, let us consider $M=15,31$ and $K=6$. In these cases, $B_{1}=1$ and $B_{2}=2^{0}-2^{-6}$ are used, according to Table 3. The proposed compensator needs 10 adders. The overall gain responses along with the passband zooms are shown in Fig. 4, featuring desirable filter performance.

\section{COMPARISON WITH OTHER METHODS}

In this section the proposed compensator structure implemented with the coefficients in Table 3, is compared with some proposed compensators proposed so far. The main 
features obtained by the methods considered in this comparison are summarized in Table 4 and discussed below.

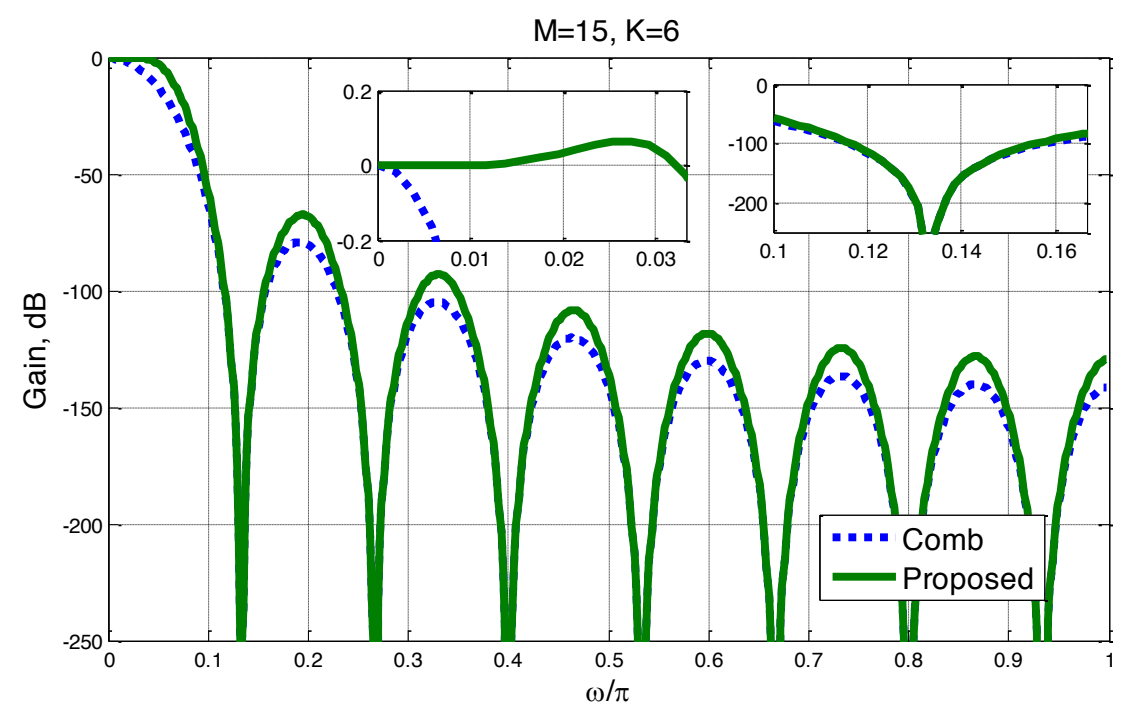

(a) $M=15, K=6, B_{1}=1$ and $B_{2}=2^{0}-2^{-6}$.

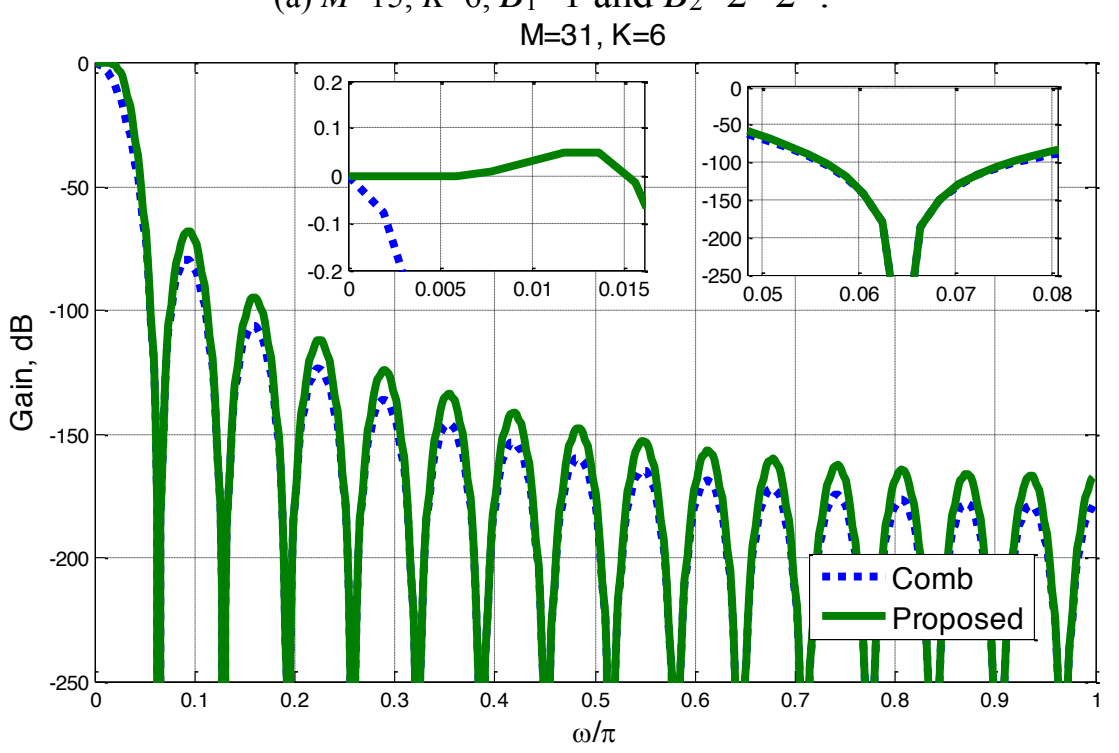

(b) $M=31, K=6, B_{1}=1$ and $B_{2}=2^{0}-2^{-6}$.

Fig. 4. Illustration of the proposed compensator for $K=6$ and two different values of $M$ with coefficients from Table 3.

\subsection{Comparison with multiplierless methods}


Let us start this comparative study by considering the multiplierless methods reported in [3] and [4]. The method proposed in this paper can be viewed as an extension of the sinesquared magnitude response method reported in [3]. The method in [3] has the advantage of lower complexity e.g., requiring 3 adders for $K=1,2,4$ and 4 adders for $K=3$, and 5 . Fig. 5 compares the overall gain responses for $M=24$ and $K=4$ obtained with the method proposed in this paper and that presented in [3].

The zoom in the passband confirms that the proposed method provides better compensation with the maximum absolute passband deviation of $\mathbf{0 . 0 6 5} \mathbf{~ d B}$ in contrast with $0.34 \mathbf{~ d B}$, obtained by the compensator presented in [3].

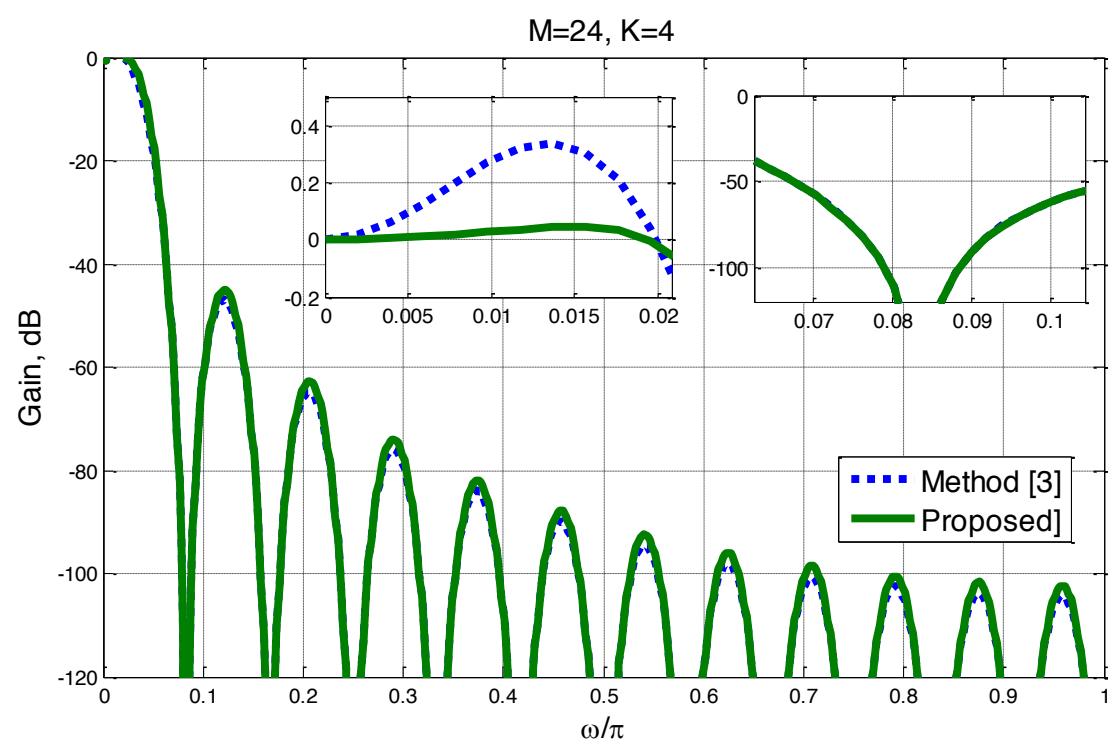

Fig. 5. Comparison with the method reported in [3].

\begin{abstract}
Moreover, there is no significant increase of the sidelobes in the proposed method, as can be observed in the zoom of the first folding band in Fig. 5.
\end{abstract}


Additionally, the advantages of both methods are that the design parameters are only defined by values of $K$, and thus it is not necessary to redesign compensation filter for new values of $M$.

The method presented in [4] uses the amplitude transformation of the cosine-squared magnitude characteristic. This method is compared in Fig. 6 with the one proposed in this work, considering $M=25$ and $K=5$. Note that the method reported in [4] has the advantage of lower complexity, requiring fewer adders (4 adders compared with 11 in the proposed method). However, as shown in the zoom in the passband, the proposed method has better compensation with an absolute value of the passband deviation of $\mathbf{0 . 0 6 5 d B}$ while in the method [4] this value is $\mathbf{0 . 4 d B}$.

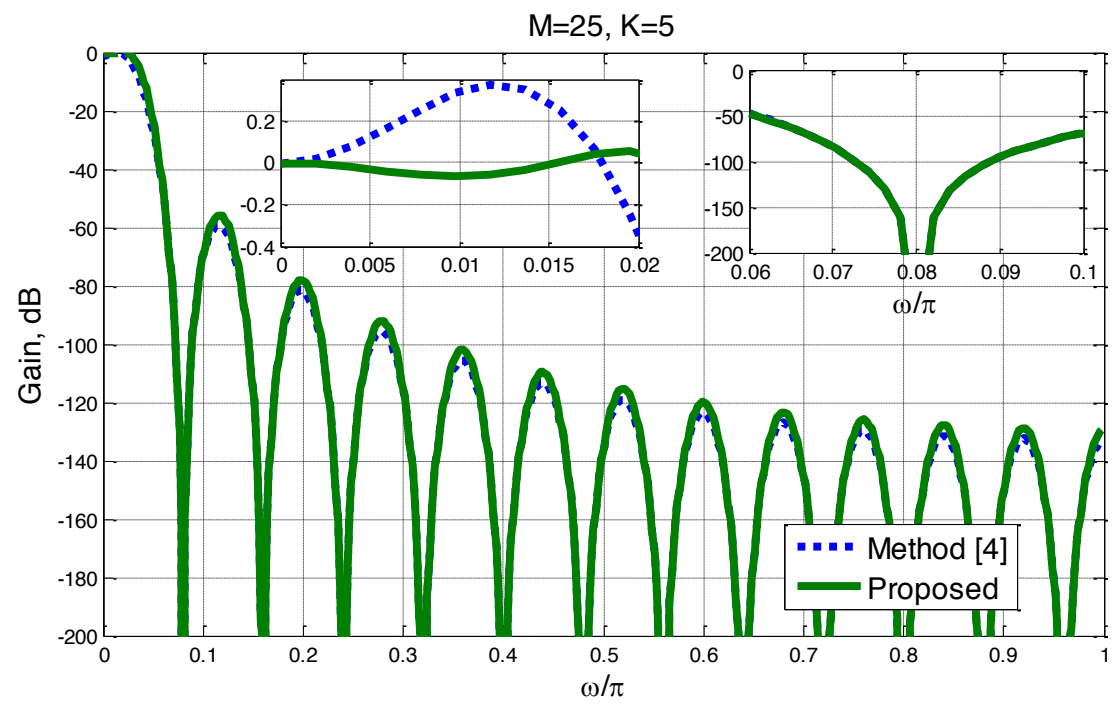

Fig. 6. Comparison with the method reported in [4].

The zoom in the first folding band indicates that the attenuations are equal in the folding bands, despite that in the proposed filter there are small increases of the sidelobes.

Another interesting approach is presented in [5], which is based on a method based on the so-called interval analysis. In this method, the compensator coefficients are expressed 
as the Sums of Powers of Two (SPT), obtained by global optimization technique, which is in turn based on the interval analysis, thus resulting in a multiplierless design [5]. The filters coefficients in the SPT forms are given in table in terms of the values of $K$ and the specified number of the compensator coefficients. The main advantage of this method is that it provides the filter coefficients not only for different values of $K$ but also for different compensator orders. The proposed compensator is compared with that of [5], with the same complexity, taking $M=32$ and $K=5$, as illustrated in Fig. 7. The compensator [5] has 5 coefficients and requires 11 adders, the same as in the proposed method. The absolute value of the maximum passband deviation in [5] is $\mathbf{0 . 1 2 5} \mathbf{~ d B}$ in contrast to $0.067 \mathrm{~dB}$ achieved by the proposed method.

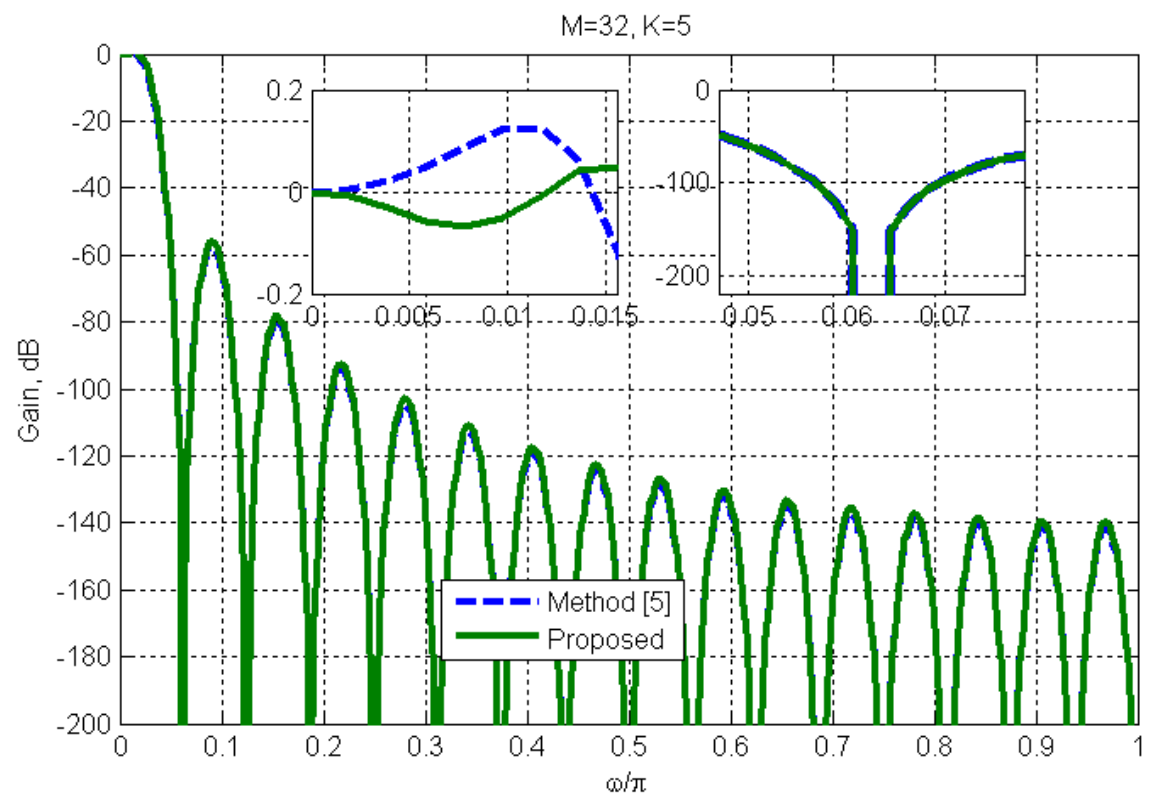

Fig. 7. Comparison with the method reported in [5].

The attenuations in the folding bands are similar in both approaches.

\subsection{Comparison with methods requiring multipliers}


We now compare the proposed method with some existing compensators implemented using multipliers. For instance, the method reported in [7] is based on maximally flat error criterion and the compensator coefficients (up to 18 coefficients) are obtained by solving a system of linear equations. Fig. 8 illustrates the compensation achieved with this method considering $M=30$ and $K=4$. As a result of this maximally-flat design, the compensated comb has a flat-magnitude characteristic in the lower frequencies of the passband, resulting in slightly better characteristic in that sub-band, when compared to the proposed method. However, the proposed method has better characteristic in the upper part of the passband. Additionally, the absolute value of the maximum passband deviation in [7], is $\mathbf{0 . 1 2 8} \mathbf{~ d B}$, which is higher than the one obtained with the proposed compensator, $0.064 \mathrm{~dB}$.

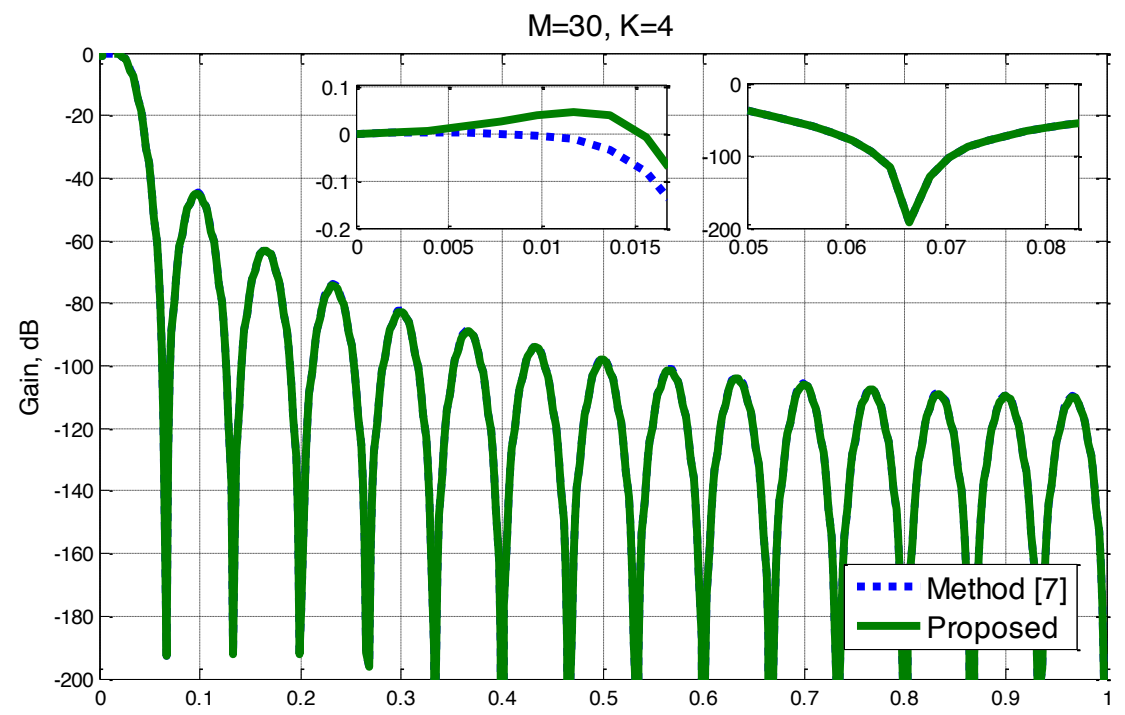

Fig. 8. Comparison with the method presented in [7].

The attenuations in the folding bands are equal for both methods. In addition, it is important to remark that the main disadvantage of method in [7], is that it requires 5 multipliers and 10 adders. 
Finally, this study is concluded by comparing the proposed design method with the method reported in [9], which is based on the use of a minimum error function. In this case, the compensator is defined by three coefficients, [-a/(1-2a), 1/(1-2a), $-a /(1-2 a)]$, where $a$ is a parameter obtained by minimizing the error function, for given $M$ and $K$. For each value of $M$ and $K$, the compensator coefficients are obtained by minimizing error functions.

Fig. 9 compares the magnitude responses of the proposed methodology with the one reported in [9], considering $M=17$ and $K=5$, (the parameter $a$ is 0.1805 ). Note that the proposed compensator provides better compensation. The absolute value of the maximum passband deviation in the method from [9] and the proposed method are respectively 0.68dB and 0.064dB. Additionally, the method from [9] needs 2 multipliers and 2 adders.

The summary of the main features of proposed method and those of [3]-[7] is presented in Table. 4.

On the basis of Table 4, and the above discussion, it can be concluded that, among the considered designs, the proposed compensator represents the best choice in terms of passband deviation and reduced hardware complexity, computed as the number of required multipliers and adders in each case. 
Table 4: Summary of the main features of proposed method and those of [3]-[7].

\begin{tabular}{|c|c|c|c|c|c|c|}
\hline $\begin{array}{c}\text { Compensa } \\
\text { tor }\end{array}$ & $\begin{array}{c}\text { Coefficie } \\
\text { nts given in } \\
\text { Table }\end{array}$ & $\begin{array}{l}\text { Max flat } \\
\text { design }\end{array}$ & $\begin{array}{c}\text { Max } \\
\text { absolute } \\
\text { deviation }\end{array}$ & $\begin{array}{c}\text { Number } \\
\text { of } \\
\text { multipliers }\end{array}$ & $\begin{array}{c}\text { Max } \\
\text { number of } \\
\text { adders }\end{array}$ & $\begin{array}{c}\text { Significan } \\
\text { tly affected } \\
\text { folding } \\
\text { bands }\end{array}$ \\
\hline $\begin{array}{c}\text { This } \\
\text { work }\end{array}$ & Yes & No & $<\mathbf{0 . 1 d B}$ & $\mathbf{0}$ & $\mathbf{1 1}$ & No \\
\hline Method [3] & Yes & No & $<0.3 \mathrm{~dB}$ & 0 & 4 & No \\
\hline Method [4] & Yes & No & $<0.4 \mathrm{~dB}$ & 0 & 4 & No \\
\hline Method [5] & Yes & No & $<0.2 \mathrm{~dB}$ & 0 & 11 & No \\
\hline Method [7] & No & Yes & $<0.2 \mathrm{~dB}$ & 5 & 10 & No \\
\hline Method [9] & No & No & $<0.7 \mathrm{~dB}$ & 2 & 2 & No \\
\hline
\end{tabular}

\section{CONCLUSIONS}

A novel comb-based multiplierless compensator filter is introduced; this filter offers good compensation in the wide passband, while achieving reduced hardware complexity compared to previous approaches. It is demonstrated that the proposed structure has the absolute passband deviation less than $0.1 \mathrm{~dB}$. The proposed compensator filter is a cascade of two simple multiplierless filters and exhibits better features than the comb compensator filters previously proposed in the literature. As compensators in general, the proposed compensator filter slightly increases side lobes in the magnitude response of comb filter without penalizing significantly the alias rejection.

\section{Acknowledgement}

This work is supported by CONACYT grant No. 179587.

\section{REFERENCES}

[1] Harris, F. J.: Multirate signal processing for communication systems, Prentice Hall, New Jersey, (2004).

[2] Hogenauer, E.: An economical class of digital filters for decimation and interpolation. IEEE Transactions Acoustic, Speech and Signal Processing, ASSP-29, 155-162, (1981). 
[3] Jovanovic Dolecek, G., Fernandez-Vazquez, A.: Trigonometrical approach to design a simple wideband comb compensator. International Journal of Electronics and Communications, 68, 437-441, (2014).

[4] Romero, D.E.T., Jovanovic Dolecek, G.: Application of amplitude transformation for compensation of comb decimation filters. Electronics Letters, 49, 985-987, (2013).

[5] Pecotic, M., Molnar, G., Vucic M.: Design of CIC compensators with SPT coefficients based on interval analysis. Proc. 35th IEEE Int. Convention MIPRO 2012, 123-128, (2012).

[6] Fernandez-Vazquez, A., Jovanovic Dolecek, G.: Maximally flat CIC compensation filter: Design and multiplierless implementation. IEEE Transactions on Circuits and Systems II: Express Brief, 54, 113-117, (2012).

[7] Molnar, G., Vucic, M.: Closed-form design of CIC compensators based on maximally flat error criterion. IEEE Transactions on Circuits and Systems II: Express Brief, 58, 926-930, (2011).

[8] Jovanovic Dolecek, G.: Simple wideband CIC compensator. Electronics Letters, 45, 1270-1272, (2009).

[9] Kim, S., Lee, W. C., Ahn, S., Cho, S.: Design of CIC roll-off compensation filter in a W-CDMA digital IF receiver. Digital Signal processing, 16, 846-854, (2006).

[10] Jovanovic Dolecek, G., Harris, F.: Design of wideband CIC compensator filter for a digital IF receiver. Digital Signal Processing, 19, 827-837, (2009).

[11] Fernandez-Vazquez, A., Jovanovic Dolecek, G.: "A general method to design GCF compensation filter. IEEE Transactions on Circuits and Systems II: Express Brief, 56, 409-413, (2009).

[12] Yeung, K. S., Chan, S. C.: The design and multiplier-less realization of software radio receivers with reduced system delay. IEEE Transactions on Circuits and Systems-I: Regular papers, 51, 2444-2459, (2004).

\section{APPENDIX}

Here, the derivation of (29) is presented in detail.

From (27), we have:

$$
\left|H_{c}\left(e^{j \omega_{k}}\right)\right|<10^{\left|\delta_{d}\right| / 40} .
$$

Using (14), we write:

$$
\left|H_{c}\left(e^{j \omega_{k}}\right)\right|=\left|H\left(e^{j \omega_{k}}\right) G_{1}\left(e^{j M \omega_{k}}\right) G_{2}\left(e^{j M \omega_{k}}\right)\right|,
$$

where $\omega_{k}$ are arbitrary frequencies in the passband, given by

$$
\omega_{k}=\frac{\pi k}{2 M N} ; k=1, \ldots N
$$


Placing (A.2) into (A.1), we get:

$$
\left|H\left(e^{j \omega_{k}}\right) G_{1}\left(e^{j M \omega_{k}}\right) G_{2}\left(e^{j M \omega_{k}}\right)\right|<10^{\left|\delta_{d}\right| / 40} .
$$

From (3), we write by noting that the comb magnitude response is positive in the passband:

$$
\left|H\left(e^{j \omega_{k}}\right)\right|=\left[\frac{1}{M} \frac{\sin \left(\omega_{k} M / 2\right)}{\sin \left(\omega_{k} / 2\right)}\right]^{K}
$$

We rewrite (A.5) as:

$$
\left|H\left(e^{j \omega_{k}}\right)\right| \approx\left[\frac{1}{M} \frac{\sin \left(\omega_{k} M / 2\right)}{\omega_{k} / 2}\right]^{K} .
$$

Placing (A.3) into (A.6) we get:

$$
\left|H\left(e^{j \omega_{k}}\right)\right| \approx\left[\frac{\sin (\pi k /(4 N))}{\pi k /(4 N)}\right]^{K} .
$$

Similarly, using (4) we write:

$$
\left|G_{2}\left(e^{j \omega_{k} M}\right)\right|=1+B_{2 s_{k}} \sin ^{2}\left(\omega_{k} M / 2\right)=1+B_{2 s_{k}} \sin ^{2}(\pi k /(4 N)) .
$$

From (A.4), (A.7) and (A.8) we arrive at:

$$
\left[\frac{\sin (\pi k /(4 N))}{\pi k /(4 N)}\right]^{K} G_{1}\left(e^{j M \omega_{k}}\right)\left[1+B_{2 s_{k}} \sin ^{2}(\pi k /(4 N))\right]<10^{\left|\delta_{d}\right| / 40}
$$

From here we have: 


$$
\left[1+B_{2 s_{k}} \sin ^{2}(\pi k /(4 N))\right]<\frac{\left[\frac{\pi k /(4 N)}{\sin (\pi k /(4 N))}\right]^{K}}{G_{1}\left(e^{j M \omega_{k}}\right)} 10^{\left|\delta_{d}\right| / 40}
$$

Also,

$$
B_{2 s_{k}} \sin ^{2}(\pi k /(4 N))<\frac{-G_{1}\left(e^{j M \omega_{k}}\right)+\left[\frac{\pi k /(4 N)}{\sin (\pi k /(4 N))}\right]^{K} 10^{\left|\delta_{d}\right| / 40}}{G_{1}\left(e^{j M \omega_{k}}\right)},
$$

and

$$
B_{2 s_{k}}<\frac{-G_{1}\left(e^{j M \omega_{k}}\right)+\left[\frac{\pi k /(4 N)}{\sin (\pi k /(4 N))}\right]^{K} 10^{\left|\delta_{d}\right| / 40}}{G_{1}\left(e^{j M \omega_{k}}\right) \sin ^{2}(\pi k /(4 N))} .
$$

Using (5) we write:

$$
\left|G_{1}\left(e^{j \omega_{k} M}\right)\right|=1+B_{1} \sin ^{2}\left(\omega_{k} M / 2\right)=1+B_{1} \sin ^{2}(\pi k /(4 N)),
$$

where values $B_{1}$ are given in Table 2 .

Finally, from (A.12) and (A.13) we arrive at:

$$
B_{2 s_{k}}<\frac{-\left[1+B_{1} \sin ^{2}(\pi k /(4 N))\right]+\left[\frac{\pi k /(4 N)}{\sin (\pi k /(4 N))}\right]^{K} 10^{\left|\delta_{d}\right| / 40}}{\left[1+B_{1} \sin ^{2}(\pi k /(4 N))\right] \sin ^{2}(\pi k /(4 N))}, k=1, \ldots, N
$$

